\title{
Possible evidence for electromagnons in multiferroic manganites
}

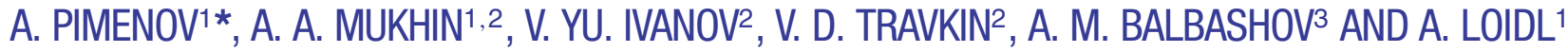 \\ ${ }^{1}$ Experimentalphysik V, Center for Electronic Correlations and Magnetism, Universität Augsburg, Universitätsstr. 2, 86135 Augsburg, Germany \\ ${ }^{2}$ A. M. Prokhorov General Physics Institute of the Russian Academy of Sciences, 119991 Moscow, Russia \\ ${ }^{3}$ Moscow Power Engineering Institute, 105835 Moscow, Russia \\ *e-mail: andrei.pimenov@physik.uni-augsburg.de
}

M agnetodielectric materials are characterized by a strong coupling of the magnetic and dielectric properties and, in rare cases, simultaneously show both magnetic and polar order. Among other multiferroics, $\mathrm{TbMnO}_{3}$ and $\mathrm{GdMnO}_{3}$ reveal a strong magneto-dielectric coupling and as a consequence fundamentally different spin excitations exist: electro-active magnons (or electromagnons), spin waves that can be excited by a.c. electric fields. Here we provide evidence that these excitations appear in the phase with an incommensurate magnetic structure of the manganese spins. In external magnetic fields this incommensurate structure can be suppressed and the electromagnons wiped out, thereby inducing considerable changes in the index of refraction from d.c. up to terahertz frequencies. Hence, besides adding a creature to the zoo of fundamental excitations, the refractive index can be tuned by moderate magnetic fields, which enables the design of the next generation of optical switches and optoelectronic devices.

Enormous progress has been made in the field of multiferroics and the discovery of new classes of ferroelectromagnets (FEMs) with the simultaneous occurrence of magnetic and polar order ${ }^{1-5}$ has triggered a revival ${ }^{6,7}$ of this old field of magneto-dielectric effects and the electrodynamics of multiferroic media ${ }^{8}$. As well as promising applications of FEMs in the field of modern electronics, for example, as multiple-state memory devices with mutual magnetic or electric control or as magnetically switchable optical devices, fascinating new problems can be tackled in basic research, such as the search for magneto-dielectric excitations. The existence of elementary excitations owing to the magnetodielectric interaction, originally termed Seignette-Magnons, was theoretically predicted 35 years ago ${ }^{9}$. Here we report the first possible observation of new hybrid excitations in $\mathrm{GdMnO}_{3}$ and $\mathrm{TbMnO}_{3}$, namely magnons, which can be excited by an a.c. electrical field and can be used to fine tune the index of refraction by moderate magnetic fields.

To demonstrate these effects we choose the two multiferroic manganites, $\mathrm{GdMnO}_{3}$ and $\mathrm{TbMnO}_{3}$. Systematic investigations of the magnetic ${ }^{10,11}$ and multiferroic properties ${ }^{1,2,12,13}$ of the rare-earth manganites $\mathrm{RMnO}_{3}(\mathrm{R}=\mathrm{Gd}, \mathrm{Tb}, \mathrm{Dy})$ have revealed a transition from a paramagnet (PM) into an incommensurate antiferromagnet (IC-AFM), and subsequently into a canted-antiferromagnetic (CA-AFM) structure in $\mathrm{GdMnO}_{3}$ or a commensurate antiferromagnetic (C-AFM) phase in $\mathrm{TbMnO}_{3}$ and $\mathrm{DyMnO}_{3}$. At the lock-in transition from the IC-AFM to the C-AFM, ferroelectricity is induced ${ }^{1,2,12}$. Neutron scattering experiments have revealed $^{14}$ that the lock-in transition in $\mathrm{TbMnO}_{3}$ rather corresponds to a transition into a non-collinear incommensurate magnetic structure.

Figure 1 represents magnetic-field-induced changes in the terahertz-dielectric properties of $\mathrm{GdMnO}_{3}$ and $\mathrm{TbMnO}_{3}$ at selected temperatures close to and below the IC-CA transition. The insets in both frames provide schematic $(B, T)$ phase diagrams for $B \| c$ (refs 11,12), where $B$ is the magnetic flux density and $T$ the temperature. One significant difference is that the CA-AFM phase in $\mathrm{TbMnO}_{3}$ is shifted to much higher fields and the low-temperature phase is a modulated magnetic and ferroelectric phase ${ }^{12,14}$. In $\mathrm{GdMnO}_{3}$ the CA-AFM extends almost to zero fields and a metastable phase exists for $9 \mathrm{~K}<T<18 \mathrm{~K}$. At the magnetic-field-induced transition from the modulated to the CA-AFM structure both the dielectric constant and the dielectric loss reveal significant step-like changes in both compounds. In $\mathrm{GdMnO}_{3}$ the transition reveals almost no hysteresis at temperatures above $30 \mathrm{~K}$, but hysteresis effects increase approaching temperatures close to $18 \mathrm{~K}$. For $9 \mathrm{~K}<T<18 \mathrm{~K}$ the IC-AFM state is not recovered after removal of the external magnetic field and the sample remains a CA-AFM. Therefore, in this range of temperatures both the CA-AFM and the modulated phase are energetically very close and are obviously in metastable equilibrium. For $T=15 \mathrm{~K}$, the dielectric permittivity $\varepsilon^{*}(B)$ is typical for this metastable range and can be obtained within a zero-field-cooling cycle. After switching to the CA-AFM state the sample remains in this state independent of the subsequent magnetic-field history. The application of a negative magnetic field can only reverse the direction of the effective magnetic moment, but no changes are observed in dielectric properties.

In order to clarify the physical mechanism of the magneticfield-induced changes in $\mathrm{GdMnO}_{3}$ and $\mathrm{TbMnO}_{3}$, we measured the 


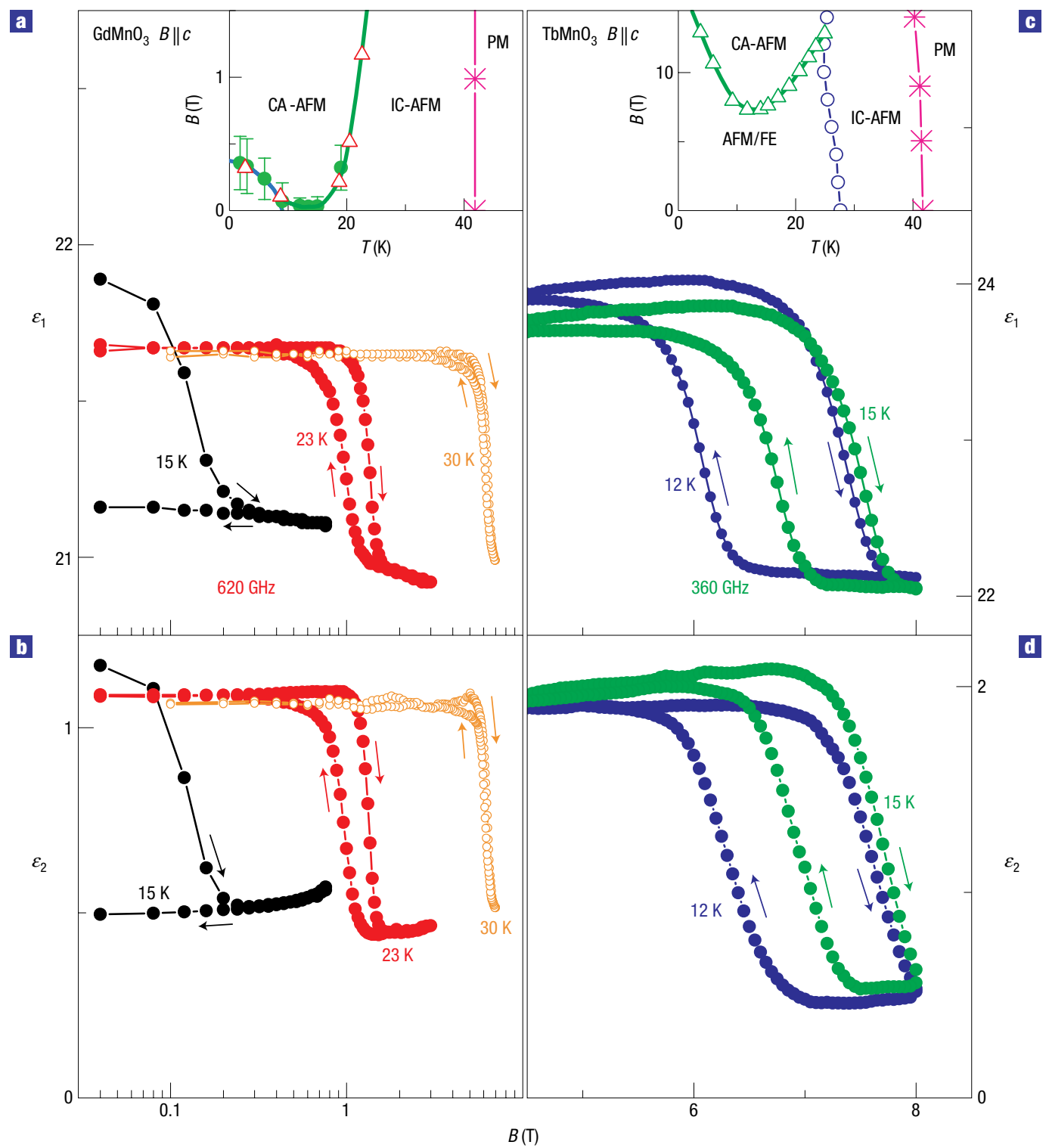

Figure 1 Terahertz-magnetodielectric effect in $\mathrm{GdMnO}_{3}$ and $\mathrm{TbMnO}_{3}$. a-d, Real $(\mathbf{a}, \mathbf{c})$ and imaginary $(\mathbf{b}, \mathbf{d})$ parts of the $a$-axis dielectric constant in $\mathrm{GdMnO}_{3}(\mathbf{a}, \mathbf{b})$ and in $\mathrm{TbMnO}_{3}(\mathbf{c}, \mathbf{d})$ as a function of external magnetic field parallel to the $c$ axis and with the a.c. electric field parallel to the $a$ axis. All data have been obtained with zero-field cooling starting from $T \geq 28 \mathrm{~K}$ (IC-AFM phase). The insets show schematically the $(B, T)$ phase diagrams of both compounds ${ }^{11,12}$. GdMn $0_{3}$ : filled circles and stars represent the results of magnetization measurements, open triangles are taken from dielectric data; the error bars reflect the difference between field-up and field-down runs; the blue line separates the complex region due to $\mathrm{Gd}$-ordering. $\mathrm{TbMnO}_{3}$ : circles, triangles and stars represent the results of dielectric, pyroelectric and magnetization measurements, respectively ${ }^{12}$.

frequency dependence of the dielectric properties both with and without external magnetic field. These results are presented in Fig. 2 and constitute the basic result of this work. The data obtained without magnetic field and with the electric a.c. component $e \| a$ show a broad relaxation-like excitation with characteristic frequency $v_{0}=23 \pm 3 \mathrm{~cm}^{-1}$ in $\mathrm{GdMnO}_{3}$ and $v_{0}=20 \pm 3 \mathrm{~cm}^{-1}$ in $\mathrm{TbMnO}_{3}$ for all temperatures. The dielectric contribution of this excitation increases with decreasing temperature and saturates in the low-temperature magnetic phase. No significant changes are observed in $\mathrm{TbMnO}_{3}$ (see inset of Fig. 1c) when passing the magnetic phase boundary at $28 \mathrm{~K}$, although the damping of the mode decreases. We recall that this low-temperature phase in $\mathrm{TbMnO}_{3}$ is ferroelectric with the polarization $P \| c$. Similar effects are observed in $\mathrm{GdMnO}_{3}$. Here the IC-AFM phase remains stable with no induced ferroelectricity. From this observation it is clear that, depending on symmetry, electromagnons can be observed in systems with inhomogeneous spin structure and magneto-dielectric coupling, independent of the existence of static ferroelectric polarization. It is unclear whether the narrowing of the excitations results from the onset of Gd-ordering $(T \approx 8 \mathrm{~K})$ in $\mathrm{GdMnO}_{3}$ and of $\mathrm{Tb}$-ordering $(T \approx 7 \mathrm{~K})$ in $\mathrm{TbMnO}_{3}$. The direct connection of the observed excitation with the magnetic subsystem can be immediately documented by applying a static magnetic field along the $c$ axis: the imaginary part of the dielectric constant (Fig. 2b,d) is suppressed by more than a factor of two and the real part (Fig. 2a,c) is reduced by about $\Delta \varepsilon_{1} \sim 2$. This simultaneous 


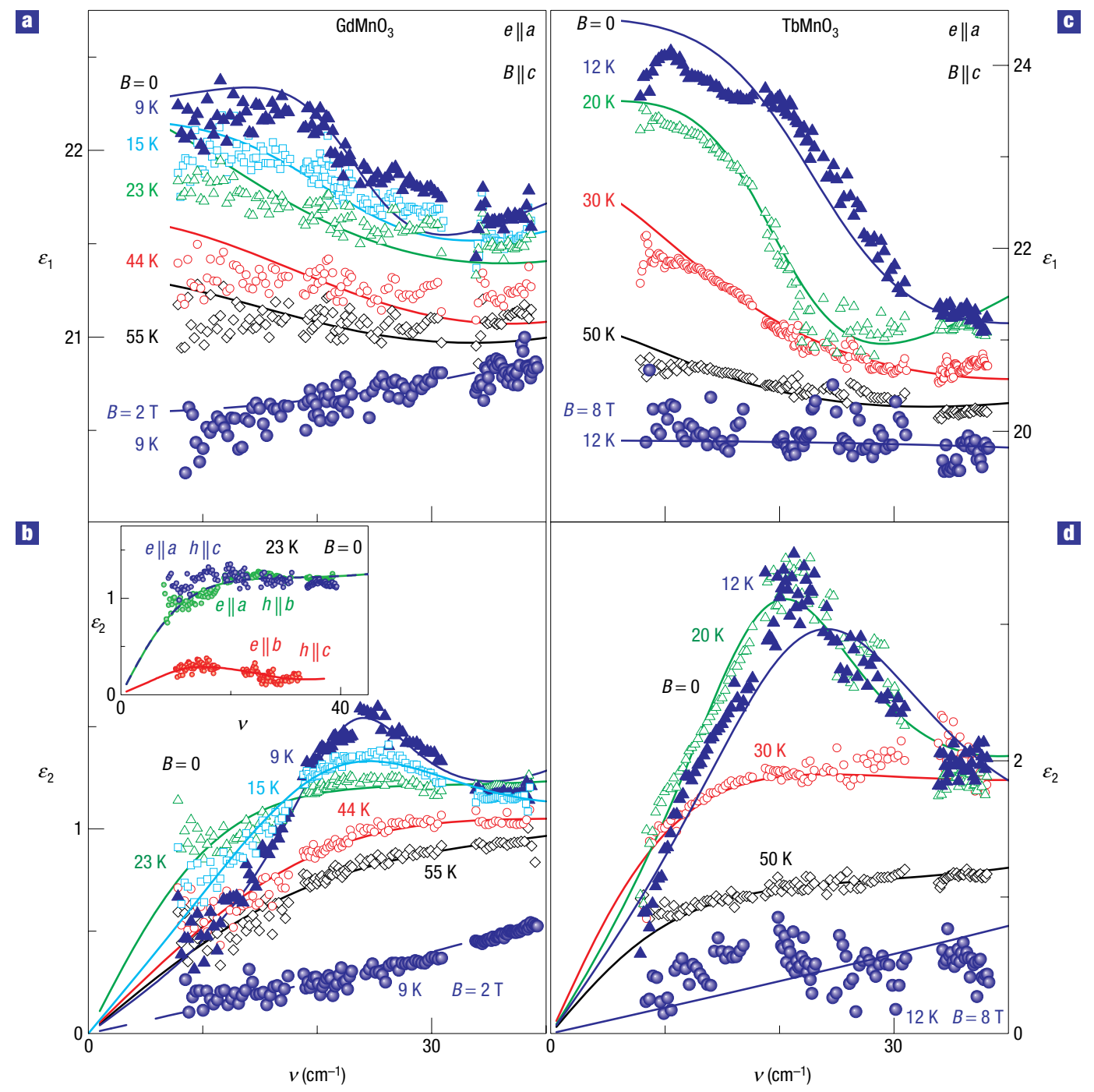

Figure 2 Spectra of electromagnons in $\mathrm{GdMnO}_{3}$ and $\mathrm{TbMnO}_{3}$. a-d, Frequency dependence of the real $(\mathbf{a}, \mathbf{c})$ and imaginary $(\mathbf{b}, \mathrm{d})$ parts of the terahertz-dielectric function in $\mathrm{GdMnO}_{3}(\mathbf{a}, \mathbf{b})$ and $\mathrm{TbMnO}_{3}(\mathbf{c}, \mathbf{d})$ with $e \| a$ and $B \| c$. Open symbols represent experimental data in zero external magnetic field and in the IC-AFM phase. Solid lines represent model calculations adding an over-damped lorentzian (dashed lines) to the residual high-frequency contribution. Filled spheres represent the data in the CA-AFM state obtained by applying $B=2 \mathrm{~T}\left(\mathrm{GdMnO}_{3}\right)$ and $B=8 \mathrm{~T}\left(\mathrm{TbMnO}_{3}\right)$ along the $c$ axis. The corresponding zero-field data are shown by filled triangles. The inset in $\mathbf{b}$ demonstrates unique ( $e \| a, h$-independent) excitation conditions for electromagnons.

suppression of $\varepsilon_{1}$ and $\varepsilon_{2}$ reflects the mutual connection of both quantities through the Kramers-Kronig relations.

The influence of an external magnetic field on the dielectric properties of $\mathrm{GdMnO}_{3}$ and $\mathrm{TbMnO}_{3}$ can be compared with the marked changes of infrared properties in colossal magnetoresistance manganites ${ }^{15}$. In the latter compounds the broad conductivity maximum at midinfrared frequencies $(\sim 0.5 \mathrm{eV})$ is transferred to the Drude-like relaxation at the metalto-insulator transition. In Ca-doped $\mathrm{PrMnO}_{3}$, in particular, a metal-to-insulator transition can be induced by magnetic fields ${ }^{15}$, which leads to magnetoresistance of several orders of magnitude. In the case of $\mathrm{GdMnO}_{3}$ and $\mathrm{TbMnO}_{3}$, the basic difference is that the transition in magnetic field takes place between two dielectric phases and only minimal changes in the low-frequency absorption are observed.

The unusual point concerning the observed mode in $\mathrm{GdMnO}_{3}$ and $\mathrm{TbMnO}_{3}$ is that it is excited by the electric a.c. component of the radiation in spite of the clear connection of this mode to the modulated magnetic structure. To prove that this spin-wave excitation depends on the a.c. electric field, and to determine the symmetry-allowed electromagnons, we performed various experiments using the radiation with the electric and magnetic components polarized along all principal crystallographic axes. An impressive example of this behaviour is documented in the inset of Fig. 2b, which shows the suppression of the magnetic excitation when the electric a.c. component is rotated from $e \| a$ to $e \| b$ leaving the magnetic field unchanged. In contrast, this excitation remains unchanged if the a.c. magnetic field $h$ is rotated from $h \| c$ to $h \| b$. This behaviour can be contrasted with the excitation of antiferromagnetic resonance modes by magnetic a.c. components only ${ }^{16,17}$. The results of the inset of Fig. $2 b$ are fully compatible with the crystal symmetry and a modulated spin structure with propagation along the crystallographic $b$ direction. The sensitivity of the incommensurate mode to the a.c. electric 
field in $\mathrm{GdMnO}_{3}$ and $\mathrm{TbMnO}_{3}$ demonstrates strong coupling of magnetic and lattice degrees of freedom, reflecting the close correlation of spin-structure and electric polarization.

Magnetodielectric (magnetoelectric) coupling plays a key role in exciting spin waves in a inhomogeneous modulated spin structure, as a homogeneous interaction is forbidden by symmetry arguments. The main contribution of this inhomogeneous magnetodielectric interaction ${ }^{18}$ to the density of the free energy is determined by $\Phi_{\mathrm{me}}=-a_{x} P_{x}\left(A_{x} \partial A_{y} / \partial y-A_{y} \partial A_{x} / \partial y\right)-$ $a_{z} P_{z}\left(A_{z} \partial A_{y} / \partial y-A_{y} \partial A_{z} / \partial y\right)$, where $\mathbf{P}$ is the electric polarization, $\mathbf{A}$ is the antiferromagnetic vector of the manganese spins and $a_{x, z}$ are magneto-dielectric interaction constants. This form of $\Phi_{\mathrm{me}}$ is obtained using transformation properties of $\mathbf{A}, \partial \mathbf{A} / \partial y$ and $\mathbf{P}$ according to eight irreducible representations (with a propagation vector $\mathbf{k}=0$ ) of the Pbnm crystallographic space group. The gradient terms are non-zero for a modulated spin structure with $\mathbf{k}=\left(0, k_{y}, 0\right)$. Including the usual dielectric contribution $\Phi_{\mathrm{E}}=-\mathbf{P E}+\mathbf{P}^{2} / 2 \chi_{\mathrm{E}}$, where $\chi_{\mathrm{E}}$ is electric susceptibility and $\mathbf{E}$ is electric field, and minimizing by $\mathbf{P}$, this magneto-dielectric interaction couples spin oscillations with the homogeneous a.c. electric field, contributes to the dielectric constant and can also induce spontaneous electrical polarization in a modulated magnetic structure. An alternative form of the magnetodielectric coupling can be obtained by expansion of the free energy by $\mathbf{P}$ and magnetic-order parameters $\sigma_{v}(k)$ of the modulated structure with $\mathbf{k}=\left(0, k_{y}, 0\right)$ using their transformation with respect to irreducible representations $\Gamma_{v}$ of the propagation vector group $G_{k}$ (ref. 14). Here spontaneous electrical polarization in a modulated magnetic structure with a strong dependence on symmetry can also be induced (for example, $P_{z} \sim\left\langle A_{z} \partial A_{y} / \partial y-A_{y} \partial A_{z} / \partial y\right\rangle_{\text {average }}$ ). Interestingly, the spin oscillations excited by the electric field correspond to non-zero wavevectors $\mathbf{k}$ of spin waves, and the resonance frequencies of electrically and magnetically excited modes are well separated in energy.

We have shown that in magneto-dielectric materials fundamentally new hybrid spin-lattice excitations exist that can be excited by a.c. electric fields. The appearance/disappearance of this new type of excitation comes along with significant changes in the index of refraction. Hence, electromagnons are not only interesting for basic research but also for the design of magnetooptic devices.

\section{METHODS}

Single crystals of $\mathrm{GdMnO}_{3}$ and $\mathrm{TbMnO}_{3}$ have been prepared by the floating-zone method with radiation heating. The samples have been characterized using X-ray, electrical, magnetic and thermodynamic measurements. The dielectric properties agree well with published results ${ }^{2,12}$.

For T-ray experiments $(0.1-1.2 \mathrm{THz})$ various plane-parallel plates were cut from the original boules each oriented perpendicular to one of the principal crystallographic axes. The typical size of the samples was $5 \times 5 \times 1 \mathrm{~mm}^{3}$. Different geometries were necessary to distinguish between electrical (that is, excited by the a.c. component of the electrical field of the electromagnetic radiation) and magnetic modes, and also to show that the observed mode of the IC-AFM structure is indeed excited by the electric component of the radiation.

The dynamic experiments in the frequency range $0.1<v<1.2 \mathrm{THz}$ were performed in transmittance experiments using a Mach-Zehnder interferometer ${ }^{19}$. This arrangement allowed the investigation of the transmittance and phase shift of the plane-parallel samples as a function of frequency, temperature and external magnetic field. Dynamic dielectric properties $\varepsilon^{*}(\nu, T, B)=\varepsilon_{1}+i \varepsilon_{2}$ were calculated from these quantities using the Fresnel optical equation for the complex transmission coefficient of the plane-parallel plate ${ }^{20}$ without additional assumptions. Temperature-dependent and magnetic-field experiments were carried out in a split-coil magnet providing magnetic fields up to $7 \mathrm{~T}$ and temperatures from $1.8 \mathrm{~K} \leq T \leq 300 \mathrm{~K}$.

Received 13 July 2005; accepted 21 December 2005; published 29 January 2006.

\section{References}

1. Kimura, T. et al. Magnetic control of ferroelectric polarization. Nature 426, 55-58 (2003).

2. Goto, T., Kimura, T., Lawes, G., Ramirez, A. P. \& Tokura, Y. Ferroelectricity and giant magnetocapacitance in perovskite rare-earth manganites. Phys. Rev. Lett. 92, 257201 (2004).

3. Hur, N. et al. Electric polarization reversal and memory in a multiferroic material induced by magnetic fields. Nature 429, 392-395 (2004).

4. Lottermoser, T. et al. Magnetic phase control by an electric field. Nature 430, 541-544 (2004).

5. Hemberger, J. et al. Relaxor ferroelectricity and colossal magnetocapacitive coupling in ferromagnetic $\mathrm{CdCr}_{2} \mathrm{~S}_{4}$. Nature 434, 364-367 (2005).

6. Fiebig, M. Revival of the magnetoelectric effect. J. Phys. D 38, R123-R152 (2005)

7. Spaldin, N. A. \& Fiebig, M. The renaissance of magnetoelectric multiferroics. Science 309, 391-392 (2005).

8. Smolenskii, G. A. \& Chupis, I. E. Ferroelectromagnets. Sov. Phys. Usp. 25, 475-493 (1982).

9. Bar'yakhtar, V. G. \& Chupis, I. E. Quantum theory of oscillations in a ferroelectric ferromagnet. Sov. Phys. Solid State 11, 2628-2631 (1970).

10. Kimura, T. et al. Distorted perovskite with $e_{g}^{1}$ configuration as a frustrated spin system. Phys. Rev. B 68, R060403 (2003).

11. Hemberger, J. et al. Complex interplay of $3 \mathrm{~d}$ and $4 \mathrm{f}$ magnetism in $\mathrm{La}_{1-\mathrm{x}} \mathrm{Gd}_{\mathrm{x}} \mathrm{MnO}_{3}$. Phys. Rev. B 70, 024414 (2004).

12. Kimura, T. et al. Magnetoelectric phase diagrams of orthorhombic $\mathrm{RMnO}_{3}(\mathrm{R}=\mathrm{Gd}, \mathrm{Tb}$, and $\mathrm{Dy})$. Phys. Rev. B 71, 224425 (2005)

13. Arima, T. et al. Magnetic-field-induced transition in the lattice modulation of colossal magnetoelectric $\mathrm{GdMnO}_{3}$ and $\mathrm{TbMnO}_{3}$ compounds. Phys. Rev. B 72, R100102 (2005).

14. Kenzelmann, M. et al. Magnetic inversion symmetry breaking and ferroelectricity in $\mathrm{TbMnO}_{3}$. Phys. Rev. Lett. 95, 087206 (2005)

15. Okimoto, Y. \& Tokura, Y. Optical spectroscopy of perovskite-type manganites. J. Supercond. 13, 271-284 (2000).

16. Mukhin, A. A., Biberacher, M., Pimenov, A. \& Loidl, A. Antiferromagnetic resonances and magnetization of a canted antiferromagnet. J. Magn. Reson. 170, 8-14 (2004).

17. Ivannikov, D. et al. High-field ESR spectroscopy of the spin dynamics in $\mathrm{La}_{1-\mathrm{x}} \mathrm{Sr}_{\mathrm{x}} \mathrm{MnO}_{3}(\mathrm{x} \leq 0.175)$ Phys. Rev. B 65, 214422 (2002).

18. Bar'ykhtar, V. G., L'vov, V. A. \& Yablonskii, D. A. Theory of inhomogeneous magnetoelectric effect. JETP Lett. 37, 565 (1983).

19. Kozlov, G. V. \& Volkov, A. A. in Millimetre and Submillimetre Wave Spectroscopy of Solids (ed. Grüner, G.) (Springer, Berlin, 1998).

20. Born, M. \& Wolf, E. Principles of Optics (Pergamon, Oxford, 1986).

\section{Acknowledgements}

Stimulating discussions with J. Hemberger, M. Kenzelmann and P. Lunkenheimer are gratefully acknowledged. We thank T. Kimura for sharing the data and samples of $\mathrm{TbMnO}_{3}$ with us and A. Pimenova for performing the magnetization experiments. This work was supported by BMBF (13N6917/0-EKM), by DFG (SFB484-Augsburg) and by RFBR (03-02-16759, 06-02-17514).

Correspondence and requests for materials should be addressed to A.P.

Competing financial interests

The authors declare that they have no competing financial interests.

Reprints and permission information is available online at http://npg.nature.com/reprintsandpermissions/ 